\title{
Nulla accommodatio: la razón y la fe en la hermenéutica de Spinoza
}

\section{Nulla accommodatio: reason and faith in Spinoza's hermeneutics}

\author{
Mario Narváez*
}

Resumen: En su famoso Tratado Teológico-Político, Spinoza ofrece un método de interpretación de las Escrituras con la finalidad de que, a través de su aplicación, el lector pueda capturar objetivamente el sentido de los textos y de ese modo no incurrir en el mismo error que los teólogos y filósofos cristianos o judíos que confunden el ámbito de la fe con el ámbito de la razón. Ahora bien, en la medida en que leemos los textos sagrados y los interpretamos utilizando el método propuesto por Spinoza, también estamos utilizando la razón. De este modo, cabe preguntarse -siguiendo a Zac- ¿cómo es posible que aun utilizando un método elaborado por la razón se puedan mantener separados el dominio de la razón y la fe sin que esto implique una invasión del primero sobre el segundo? Así, nuestro objetivo en el presente trabajo es examinar el modo en que Spinoza entiende que se realiza la aplicación de la razón en el proceso hermenéutico. Con vistas a este fin, luego de presentar los rasgos generales del método hermenéutico spinoziano, examinaremos críticamente la lectura propuesta por Zac, lo cual nos servirá como trampolín para presentar nuestra propia interpretación.

Palabras clave: método hermenéutico, razón, fe, Escrituras, Spinoza

Abstract: In his famous Tractatus Theologico-Politicus, Spinoza offers a method of interpreting the Scriptures so that, through its application, the reader can objectively capture the meaning of the texts and thus not incur the same error as

\footnotetext{
* Profesor y licenciado en Filosofía por la Universidad Nacional de La Plata, Argentina. Se encuentra realizando el Doctorado en la Universidad Nacional del Sur, para el cual contó con las becas I y II del CONICET. Se especializa en temas de filosofía moderna, epistemología y lógica. Es profesor adjunto en las cátedras de Filosofía y Problemas de Filosofía Contemporánea y Jefe de Trabajos Prácticos en la cátedra de Epistemología en la Universidad Nacional de La Pampa. Dirección electrónica: narvaezmario2003@hotmail.com
} 
Christian or Jewish theologians and philosophers who confuse the field of faith with the realm of reason. Now, to the extent that we read the sacred texts and interpret them using the method proposed by Spinoza, we are also using reason. In this way, we can ask ourselves -following Zac- how is it possible that, even using a method developed by reason, the domain of reason and faith can be kept separate without this implying an invasion of the former over the latter? Thus, our objective in the present work is to examine the way in which Spinoza understands that the application of reason is made in the hermeneutical process. With a view to this end, after presenting the general features of the Spinozian hermeneutical method, we will critically examine the reading proposed by Zac, which will serve as a springboard to present our own interpretation.

Keywords: hermeneutical method, reason, fait, Scriptures, Spinoza

\section{Introducción}

En su famoso Tratado Teológico-Político', Spinoza ofrece un método de interpretación de las Escrituras con la finalidad de que, a través de su aplicación, el lector pueda capturar objetivamente el sentido de los textos y de ese modo no incurrir en el mismo error que los teólogos y filósofos cristianos o judíos que confunden el ámbito de la fe con el ámbito de la razón. Esto es, el error de falsear el sentido de las Escrituras aplicando a ellas teorías de la razón o, viceversa, aplicando a la razón prejuicios de las Escrituras. Spinoza sabe que, de esta manera, también se evitará la intromisión de la religión en el terreno de la filosofía. En última instancia lo que está en juego es la libertad de filosofar ${ }^{2}$ y, a través de la

${ }^{1}$ En adelante esta obra se indicará TTP y número de página. Para la Ética, E seguido del libro en números romanos y, según el caso, $\mathrm{p}$ (proposición), dem. (demostración), cor. (corolario), esc. (escolio), def. (definición), ax. (axioma), pos. (postulado), Ap. (apéndice). En todos los casos se incluye a continuación la indicación del libro y la página en la edición canónica de Gebhardt: Spinoza, Baruch; Opera, edición de Carl Gebhardt, 4 vols., Heidelberg, 1925, reed. 1972 (G).

${ }^{2}$ Strauss, Leo, Spinoza's Critique of Religion, New York, Schoken Books, 1965, p. 258. 
"democratización" de la razón que ella implica, un mejoramiento de la sociedad en general. ${ }^{3}$

Ahora bien, en la medida en que leemos los textos sagrados y los interpretamos utilizando el método propuesto por Spinoza es claro que de algún modo también estamos aplicando la razón. En este sentido, en el siglo pasado se lo ha acusado a Spinoza de imponer, como abanderado de la Ilustración, el prejuicio racionalista, es decir, el prejuicio contra todos los prejuicios. ${ }^{4}$ Sea como fuere, el hecho es que Spinoza quiere evitar los prejuicios y, desde su punto de vista, hay una diferencia importante entre su manera de interpretar la Biblia y la de otros filósofos y teólogos que han pretendido adaptar el contenido de los textos sagrados a las enseñanzas de la razón.

De este modo, cabe preguntarse -siguiendo a Zac- ¿cómo es posible que aun utilizando un método elaborado por la razón se puedan mantener separados el dominio de la razón y el de la fe sin que esto implique una invasión del primero sobre el segundo? Esta pregunta conduce, a su vez, a esta otra, ¿cuál es la naturaleza de la aplicación de la razón en el método hermenéutico elaborado por Spinoza? La respuesta de Zac -que como

3 Yovel, Yirmiyau, "Bible Interpretation as Philosophical Praxis: A Study of Spinoza and Kant", Journal of the History of Philosophy, Vol. 11, num. 2, April 1973, p. 192.

${ }^{4}$ Gadamer, Hans Georg, Verdad y Método, Salamanca, Sígueme, 1991, p. 337. La hermenéutica presentada por Spinoza sin dudas ha sido muy importante y ha tenido mucha repercusión, aunque ciertamente no puede considerárselo el fundador de la hermenéutica científica (Para un breve examen de la cuestión de los posibles antecedentes de Spinoza véase Curley, Edwin, "Notes on a Neglected Masterpiece (I): Spinoza and the Science of Hermeneutics," in Hunter, Graeme (ed.), Spinoza: The Enduring Questions, Toronto, University of Toronto Press, 1994, pp. 65 y ss.). No obstante, como sugiere Popkin, "Spinoza comenzó un modo muy diferente de evaluar y examinar los textos de las Escrituras respecto del empleado por sus antecesores. El literalismo y contextualismo conducen a una lectura completamente secular de la Biblia". (Popkin, Richard, "Spinoza and Bible Scholarship", en: Garrett, Don (ed.), The Cambridge Companion to Spinoza, Cambridge, Cambridge University Press, 1996, p. 396). 
mostraremos es insatisfactoria- apela a una supuesta distinción en el seno de la filosofía de Spinoza entre la razón -entendida como un sistema filosófico- y el juicio crítico -encargado de llevar a cabo una serie de procedimientos metodológicos.

Nuestro objetivo es examinar el modo en que Spinoza entiende que se realiza la aplicación de la razón en el proceso hermenéutico, lo cual, creemos, nos ayudará a comprender con mayor profundidad la naturaleza del método hermenéutico del TTP. Con vistas a este fin, la discusión de la lectura de Zac nos servirá como trampolín para presentar nuestra propia interpretación. El recorrido que realizaremos es el siguiente. En primer lugar, expondremos los rasgos más generales del método hermenéutico a partir de un examen de los principios que operan en él. En segundo lugar, presentaremos la lectura de Zac en relación con el problema planteado. Luego, señalaremos los defectos que encontramos en dicha lectura e indicaremos una visión alternativa. Finalmente, presentaremos las conclusiones.

\section{El método hermenéutico de Spinoza}

Al comienzo del capítulo séptimo del TTP, Spinoza afirma que el método de interpretar la Escritura no es diferente del método de interpretar la naturaleza, el cual es entendido -bajo la influencia de Bacon-como la recopilación de datos (elaboración de "historias") y la obtención de definiciones. ${ }^{5}$ Dicho método tendría como finalidad alcanzar conocimiento

5 Spinoza, Baruch, Tratado Teológico-Político. Traducción de Atilano Domínguez. Madrid, Altaya, 1999, cap. 7, p. 193 (véase nota 151). Para un examen más preciso de la influencia de Bacon, vid. Gottlieb, Michah, "Spinoza`s Method(s) of Biblical Intepretation Reconsidered", Jewish Studies Quarterly, Vol. 14, N 3, 2007, pp. 294 y ss. Dicho autor señala que a pesar de que generalmente se ha enfatizado la influencia de Bacon sobre el método hermenéutico de Spinoza, también hay en él elementos del método cartesiano. Se refiere, fundamentalmente, al criterio de claridad y distinción. En efecto, Spinoza afirma que no debemos aceptar como 
cierto y confiable acerca del pueblo hebreo, de la vida de los profetas y fundamentalmente del contenido de los libros de las Escrituras. Sin embargo, a diferencia del método científico, el método hermenéutico no tiene como finalidad una verdad acerca de la naturaleza, sino el verdadero sentido del texto. Esto es, no se trata de juzgar si lo que dicen las Escrituras es verdadero o falso sino de comprender correctamente qué se dice. ${ }^{6}$

Ahora bien, además de los procedimientos mencionados, hay tres principios que completan el método y orientan la práctica. En primer lugar, el principio capital de la sola Scriptura -de naturaleza hermenéutica-, según el cual, todo el conocimiento para la interpretación debe ser tomado solo de las Escrituras, sin recurrir a otras fuentes, ideas o cualquier otro tipo de información que no sea la que contienen los libros sagrados. ${ }^{7}$ En segundo lugar, tenemos que mencionar un principio lógico de coherencia. Este principio implica no aceptar ninguna afirmación que no sea coherente o compatible con la doctrina más universal que pueda hallarse en las Escrituras. Ahora bien, para ello es necesario que el intérprete conozca, por así decir, el núcleo duro de las mismas, esto es, las enseñanzas más generales sobre las que todo el mundo estaría de acuerdo. En efecto, Spinoza sostiene que hay una enseñanza básica, común a todos los libros tanto del Antiguo como del Nuevo Testamento, que es clara y perceptible para cualquier lector, por más inculto que sea. A este contenido debe atenerse la interpretación subsiguiente. Admitida la existencia de estas verdades básicas, el procedimiento que recomienda Spinoza es comparable al que debe seguirse en la investigación de la naturaleza, es decir, descender de las leyes más universales y comunes (en el caso de la física, leyes relativas al movimiento y al reposo, derivadas de las nociones comunes) hacia el

doctrinas de la Escritura más que aquellas que pueden derivarse de ella con la mayor claridad posible (Ibid., p. 295).

6 "De solo enim sensu orationum, non autem de earum veritate laboramus" (TTP, p. 196, G III 100).

${ }_{7}^{7}$ TTP, p. 194, G III 99. Para los usos anteriores del principio de sola Scriptura y los antecedentes de la hermenéutica de Spinoza en general, véase Grondin, Jean, Introducción a la Hermenéutica Filosófica, Barcelona, Herder, 1999, esp. caps. 1 y 2. 
conocimiento de las cosas particulares. ${ }^{8}$ Una vez determinado el núcleo duro de la doctrina, y solamente si aparecen expresiones particulares que sean incompatibles o contradictorias con la doctrina básica, el intérprete procurará realizar, o bien una lectura metafórica, o bien una lectura en base a circunstancias históricas. Por ejemplo, para Spinoza, la afirmación según la cual Moisés mandó hacer justicia a través de la ley del talión, contradice la afirmación según la cual Jesús mandó a tolerar la injusticia y poner la otra mejilla. Tal contradicción, a su entender, se explica por la razón de que Jesús vivió en tiempos de agitación y violencia en los que convenía mantener una disposición de ánimo serena. Esto es, su objetivo, a diferencia del objetivo de Moisés, no era establecer leyes para el Estado. De otro modo, su aceptación de la injusticia resultaría contradictoria con las máximas generales de la Escritura. ${ }^{9}$

Finalmente, vemos que dentro del principio de coherencia opera, al mismo tiempo, un tercer principio, una especie de principio epistemológico de orden, que hace referencia a las dos direcciones que debe seguir la investigación. La primera etapa, que utiliza los procedimientos de la inducción baconiana, debe proceder de lo particular a lo general. Esto es, a partir de la elaboración de la historia de la Escritura se debe establecer la doctrina más general y universal. La segunda etapa, por otra parte, consiste en buscar doctrinas de menor grado de universalidad. Spinoza, afirma que tales doctrinas "brotan como riachuelos de esa doctrina universal". A esto hay que agregar que toda oscuridad o ambigüedad debe resolverse en función de ella. ${ }^{10}$ En consecuencia, resulta claro que se trata de una vía que desciende, sacando conclusiones, de lo general a lo particular. ${ }^{11}$ Spinoza cree, además, que se trata de un camino que va de lo más claro y fácil de conocer hacia lo más oscuro y confuso. "La naturaleza y la virtud de este

\footnotetext{
8 TTP, p. 199, G III 103.

9 TTP, pp. 200-201, G III 104-105. También se ve aquí como entra en juego el conocimiento histórico que permite determinar cuáles eran las intenciones de Moisés y de Jesucristo.

10 T'TP, p. 199, G III 103.

11 También en el caso de la interpretación de la mente de los profetas se debe proceder en esta dirección. TTP, p. 202, G III 106.
} 
método consisten, principalmente, en efecto, en deducir y concluir, por legítima consecuencia, las cosas oscuras a partir de las conocidas o concedidas como tales". ${ }^{12}$

En cierto modo, pareciera que Spinoza piensa aquí en la síntesis, que extrae conclusiones a partir de principios claros y evidentes. No es casual, pues, que utilice en este punto a la geometría de Euclides como ejemplo de lo que es comprensible y fácil de expresar en cualquier idioma y le atribuya la misma claridad y las mismas posibilidades de expresión al núcleo duro de la enseñanza moral de la Escritura. ${ }^{13}$

Ahora bien, como es sabido, la intención de Spinoza es interpretar los textos sagrados evitando lecturas que pongan en ellas prejuicios religiosos o filosóficos. En virtud de este objetivo es que se opone a cualquier condicionamiento o aplicación -la palabra que usa Spinoza es accommodatio- que opere, o bien adaptando las enseñanzas de las Escrituras a la razón o bien, contrariamente, sometiendo la razón a las Escrituras ${ }^{14}$. Esta idea queda claramente reflejada en su crítica a Maimónides ${ }^{15}$ y en su crítica a Alfakar, ${ }^{16}$ en tanto representantes respectivamente de una y otra posición. Así, su método permitiría una lectura -por así decir- neutral, capaz de "conocer con certeza la mente de la Escritura sobre las cosas relativas a la salvación y necesarias para la beatitud". ${ }^{17}$

\footnotetext{
12 'TTP, p. 211, G III 111.

13 TTP, pp. 210-211, G III 111-112. Hasta aquí hemos dado una descripción del método tal como lo propone Spinoza. Por razones de espacio no entraremos en la discusión de en qué medida nuestro filósofo cumple en la práctica con los preceptos de su propio método (para ello véase Gottlieb, "Spinoza`s Method(s) of Biblical Intepretation Reconsidered", pp. 302 y ss.; Yovel, "Bible Interpretation as Philosophical Praxis: A Study of Spinoza and Kant”, p. 204).

14"Primus, qui inter Pharisaeos apertè statuit Scripturam rationi esse accommodandam, fuit Maimonides... R. Jehudae Alpakhar, qui errorem Maimonidis vitare cupiens in alterum ei contrarium incidit." (TTP, p. 319, G III 180-181).

15 T'TP, pp. 212-219, G III 112-118.

16 TTP, pp. 318 y ss., G III 180 y ss.

17 TTP, p. 211, G III 111.
} 


\section{La lectura de Zac: el buen sentido y el sano juicio}

Ante este planteo, cabe preguntarse: si el reino de la razón y el de la teología deben mantenerse separados, ¿cómo es que Spinoza nos pide que utilicemos la razón para interpretar la Escritura? ¿La interpretación de Spinoza no es también una aplicación de la razón a la Escritura y, en este sentido, como afirma Zac, no corre el peligro de cometer él mismo el error que encuentra en Maimónides?18 A nuestro entender, la pregunta de Zac indica un problema muy interesante, aunque su respuesta al mismo no nos parece del todo correcta. La solución que Zac propone tiene dos partes. En primer lugar, una distinción entre dos significados del término razón en Spinoza. En segundo lugar, la adopción de uno de estos sentidos como correspondiente al método de interpretación y, a partir de allí, la exposición de las dos funciones principales de la razón, una crítica y polémica -en contra de los prejuicios- y otra, por así decir, lógica, encargada de buscar establecer la coherencia en las enseñanzas.

En lo que sigue examinaremos críticamente la interpretación de Zac. La discusión nos servirá como punto de partida para exponer nuestra visión del tema. Pues bien, según Zac, Spinoza distinguiría entre "razón", entendida como sinónimo de un sistema de ideas verdadero, es decir, como una filosofía verdadera -sentido que, a su modo de ver, referiría exclusivamente a la Ética- y otro sentido más general, menos preciso y de uso corriente, sinónimo de un cierto "buen sentido" (bon sens). Esta última acepción del término, según él, podría considerarse muy cercana a la noción de juicio y no implicaría la idea de conocimiento demostrativo; podría resumirse en la expresión "juicio crítico". ${ }^{19}$ De este modo, según Zac, la propuesta metodológica de Spinoza consistiría en la aplicación de la razón en el sentido de una crítica, de un juicio crítico, etc., mas no de una

18 Zac, Sylvain, Spinoza et l'interprétation de l'Écriture, Paris, Presses Universitaires de France, 1965, pp. 124-125.

19 "Spinoza emplea la palabra "razón" -afirma Zac- tanto en el mismo sentido que en la Ética, al hacer alusión a su propia filosofía, cuanto en un sentido más amplio que se acerca mucho a aquel que el lenguaje común da a esa palabra” (Ibid., p. 126). 
adaptación del sentido de la Escritura a la filosofía, que es lo que, a los ojos de Spinoza, hace Maimónides. Este último aspecto de la interpretación de Zac en cierto sentido es correcto, más no la distinción sobre la que se apoya, la cual no tiene suficiente base textual. En efecto, Zac en ningún momento recurre a citas textuales precisas y evita, por ejemplo, la definición de conocimiento racional de la Ética (E II, p 40, esc, G II 122). Es cierto que Spinoza menciona el sano judicio en el capítulo XV del TTP, pero esto no parece suficiente para considerarlo como el tipo de racionalidad que rige el método hermenéutico.

Para determinar el valor de esa expresión, que es la clave de la propuesta de Zac, veamos más de cerca en qué contexto aparece. La cuestión que se plantea a partir de la segunda mitad del capítulo XV se refiere a aquello que, para Spinoza, es el fundamento de la revelación y de toda la teología. Allí, nuestro filósofo se pregunta, ¿si no podemos demostrar matemáticamente el dogma según el cual la obediencia es la base de la salvación, entonces, por qué lo aceptamos? En este contexto, Spinoza responde que dicho dogma no puede ser conocido por la luz natural, es decir, demostrado matemáticamente, sino que se nos manifiesta con una certeza moral -morali certitudine - y lo aceptamos por nuestro sano judicio. Ahora bien, es evidente que el sano judicio no lleva a cabo ninguna interpretación, sino que tiene que ver con reconocer la conveniencia del fundamento teológico. El pasaje parece referirse a una cierta disposición de ánimo, a una cierta prudencia. Siendo así, como veremos más en detalle a continuación, no tiene nada que ver con el conocimiento racional tal como lo define Spinoza, esto es, como una manera de concatenar las ideas claras y distintas que surge de una determinación interna del alma, opuesta a la forma pasiva propia del conocimiento imaginativo. ${ }^{20} \mathrm{Zac}$, por el contrario, hace de este sano judicio una especie de facultad racional, a la que le atribuye todas las tareas cognoscitivas de la interpretación, esto es, combatir los

${ }^{20}$ Spinoza, Baruch, Ética demostrada según el orden geométrico, traducción de Atilano Domínguez, Madrid, Trotta, 2000. E II, p 29, esc., G II 114; TTP p. 161-162, G III 76-77. 
prejuicios, detectar incoherencias, deducir de los dogmas de la fe a partir del fundamento de la obediencia a Dios, etc. ${ }^{21}$

\section{Problemas en la lectura de Zac y propuesta superadora}

Ahora bien, en nuestra opinión, teniendo en cuenta la definición de conocimiento racional de la Ética se puede mostrar que no hay en Spinoza dos concepciones diferentes de la razón -aunque a veces utilice el término en un sentido vago, quizás cercano al uso corriente- y que dicha definición correspondería a una concepción de la razón presente tanto en la Ética como en el método de interpretación propuesto en el TTP. Veamos, en primer lugar, el pasaje en cuestión, y luego si la concepción de la razón allí expresada corresponde o no a la noción de racionalidad implicada en el método hermenéutico. Spinoza define el conocimiento racional del siguiente modo: [se da en nuestra alma una percepción]... A partir... de que tenemos nociones comunes e ideas adecuadas de las propiedades de las cosas... a éste [género de conocimiento] le llamaré razón o conocimiento de segundo género". ${ }^{22}$

Como puede colegirse a partir de aquí, para nuestro filósofo la razón o, mejor dicho, el conocimiento racional, en su sentido más cabal, no es necesariamente un sistema filosófico en particular, ni algo así como lo que hoy llamaríamos "juicio crítico" en un sentido amplio, aunque en ambos pueda haber algo de esta racionalidad que hallamos en esta definición. En cambio, tal como muestra la definición citada, y como se desprende de otros textos del corpus, conocimiento racional es aquel que parte de nociones comunes o ideas adecuadas de propiedades generales y, a través de deducciones, extrae de ellas la información necesaria para conocer

${ }^{21}$ Zac, Spinoza et l'interprétation de l'Écriture, p. 131 y ss.

22 "Denique ex eo, quod notiones communes rerumque proprietatum ideas adaequatas habe|mus...; atque hunc rationem et secundi generis cognitionem vocabo" (E II, p 40, esc. 2, G II 122). 
determinado género de objetos. ${ }^{23}$ De aquí surge un ordenamiento de las ideas adecuadas que Spinoza suele oponer al orden imaginativo que sigue pasivamente los afectos del cuerpo. ${ }^{24}$ Ciertamente, la aplicación de la razón da lugar a una filosofía y el mismo Spinoza cree que su filosofía -expuesta en la Ética - es el resultado del uso de la razón. Con todo, ello no implica una identificación de la razón con la filosofía. La razón, como veremos, debe entenderse en un sentido, por así decir, procedimental. Por lo demás, es claro que la definición citada no se corresponde con la distinción propuesta por Zac.

Pues bien, teniendo esto en cuenta y a fin de explicar el modo en que se "aplica" la razón a la interpretación de las Escrituras, en nuestra opinión, debemos distinguir dos sentidos en el uso del término "razón” por parte de Spinoza, pero de una forma casi opuesta a la sugerida por Zac. Veámoslo de la siguiente manera. Spinoza critica a Maimónides por hacer de la teología sierva de la razón, esto es, como muestran los ejemplos, por el hecho de que explica metafóricamente las historias de las Escrituras que no concuerdan con el aristotelismo y, así, puede adaptarlas a dicho sistema filosófico. Ahora bien, para Spinoza, al hacer esto Maimónides no aplica la razón a las Escrituras, sino que procede de una forma perjudicial, inútil y absurda. ${ }^{25}$ Por un lado, el procedimiento no es racional; por otro, la propia filosofía de Maimónides - el aristotelismo- tampoco lo es: basta considerar la crítica spinoziana a las nociones universales para darse cuenta de que difícilmente el aristotelismo formaría parte del conocimiento racional strictu sensu. ${ }^{26}$ Aunque lo importante para Spinoza es lo primero, es decir, que no es lícito interpretar las Escrituras desde un sistema filosófico o un sistema de la naturaleza - por más racional que sea- pues confundimos dos cosas completamente distintas, para nuestro propósito el segundo aspecto de la crítica también es importante. En efecto, sin perder de vista la definición

\footnotetext{
${ }^{23}$ Acerca de las nociones vid. E II p 37, p 38, p 39, p 40, G II 119-121. Para una discusión del tema puede consultarse la exposición de Nadler, Steven, Spinoza's Ethics. An Introduction, New York, Cambridge University Press, 2006, pp. 174-176.

${ }^{24}$ E II p 29 esc. 2; G II 114-115; TTP, p. 185, G III 93.

25 T'TP p. 217, G III 116.

${ }^{26}$ E II p 40 esc 1, G II 121.
} 
del conocimiento racional antes citada, el hecho de que Spinoza llame "racional" a la filosofía de Maimónides, nos lleva a pensar que el uso que hace Spinoza aquí del término "razón” podría considerarse un sentido débil del mismo. El término se usaría de esta manera para designar a cualquier sistema o doctrina filosófica, que pretenda pasar por racional o que haya sido considerada racional en algún momento, sin importar si es la filosofía verdadera o si no lo es.

Por otra parte, strictu sensu, dicho término correspondería, tal como lo expresa la definición que citamos arriba, a un procedimiento que parte de nociones comunes e ideas adecuadas de las cosas y deduce de allí nuevas ideas siguiendo el orden del entendimiento. ${ }^{27}$ En este último sentido, tanto el conocimiento de la naturaleza en sentido amplio (la Ética), como la geometría de Euclides o la interpretación de las Escrituras proceden racionalmente. En efecto, desde esta perspectiva la razón no tiene un contenido determinado, sino que es más bien un método o una manera de ordenar el conocimiento, que comienza por lo más general, común y claro, y avanza desde allí hacia lo menos general o lo menos claro. Tal como hemos visto en el apartado anterior, así es como Spinoza recomienda proceder según su método de interpretación, es decir, comenzando por las ideas más claras y universales de las Escrituras. Sin embargo, es importante tener en cuenta que esto no implica mezclar los ámbitos de conocimiento e interpretar uno en función del otro. Se trata más bien de un procedimiento análogo en la naturaleza y en las Escrituras, pero no de una superposición de uno sobre otro.

Ahora se ve más claramente en qué sentido Spinoza aplica la razón a las Escrituras. Obviamente no es la adaptación de los textos a un punto de vista filosófico como lo hace Maimónides, pero tampoco es en el sentido débil y poco claro de un cierto "buen sentido", "juicio crítico" o "juicio". ${ }^{28}$

27 Téngase en cuenta que Spinoza utiliza los términos "razón” y "entendimiento" -intellectus seu ratio- de una manera prácticamente sinonímica (vid. E IV c 4, G II 267).

${ }^{28}$ Según Zac: “Cuando Spinoza habla de la 'razón', en el sentido preciso de la Ética, la asocia a la 'filosofía'; cuando habla de la razón con la que se debería interpretar 
Por cierto, la aplicación consiste en el uso de un método que coincide con la definición de razón de la Ética. Quizás se podría objetar que, puesto que Spinoza distingue entre certeza moral y certeza matemática, y dado que el conocimiento de los principios de los que parte la interpretación de las Escrituras se apoya en la certeza moral, no se trataría de la misma razón. Sin embargo, Spinoza afirma explícitamente que, en ambos casos, podemos formar un concepto claro y que, en ambos casos, dicho concepto puede captarse por el entendimiento. Alcanzar la mente de las Escrituras y la de los profetas presenta numerosas dificultades, no obstante:

... no acerca de aquellas cosas que podemos captar por el entendimiento y de las que podemos formar fácilmente un concepto claro. Pues las cosas que son por naturaleza fáciles de percibir, nunca se pueden expresar con tanta oscuridad, que no se entiendan fácilmente... Porque las verdaderas enseñanzas de la piedad se expresan con las palabras más corrientes ya que son muy comunes y no menos sencillas y fáciles de comprender. ${ }^{29}$

Como se sigue de aquí, las cosas fáciles de percibir lo son para todos los hombres, no solo para los sabios. Esto se aplica tanto a las enseñanzas morales básicas de la Escritura como a los principios de la geometría-que

las Escrituras, liga la palabra razón a la palabra 'juicio"' (Spinoza et l'interprétation de l'Écriture, p. 130). Pero la utilización de este último término que pretende Zac parece poco apropiada. En efecto, el término “juicio” en la Ética es utilizado la mayor parte de las veces como sinónimo de "considerar", esto es, para expresar de qué modo algo se le presenta a uno a la mente. Juzgar es un acto que realiza cualquier persona, es indiferente que el que juzga posea conocimiento imaginativo o conocimiento racional. Por ejemplo, vid. E IV p 19 dem, G II 224; E IV p 37 esc. 2, G II 238.

${ }^{29}$ TTP, pp. 210-211, G III 111 En este punto parece haber cierta discrepancia entre el cap. XV y el cap. VII por lo que respecta a la facultad -usando un término ajeno a Spinoza- que capta los principios. En efecto, a diferencia de lo que sucede en el cap. XV donde el sano juicio nos hace aceptar aquello que tiene una cierta certeza moral, en el cap. VII -en una nota agregada por Spinoza- aquellas nociones acompañadas de certeza moral -referidas a la piedad- son equiparadas a las nociones claras y distintas que se revelan al entendimiento o a la luz natural. (Vid. TTP, pp. 210-211, G III 111 nota de Spinoza). 
Spinoza toma como punto de comparación. Es por eso que no parece necesario distinguir entre una razón, por así decir, "demostrativa" -filosofía verdadera- y una razón "popular" - "juicio crítico"- como sucede en la interpretación de Zac. ${ }^{30}$ Además de las razones expuestas, si aceptáramos la distinción de Zac no podríamos entender por qué Spinoza afirma que el método de interpretación procede del mismo modo que el método de interpretar la naturaleza. Se comprende, entonces, que "razón" no se refiere a un contenido cognoscitivo en particular, sino más bien a una forma de proceder -desde el conocimiento más claro y universal hacia el conocimiento de las cosas particulares- que puede utilizarse en distintos ámbitos del conocimiento -no solo en el campo estrictamente filosófico.

Ahora bien, ¿piensa Spinoza que el vulgo que eventualmente utilice su método de interpretación procederá demostrativamente? En efecto, una de las razones por las que Spinoza rechaza la posición de Maimónides es que el vulgo no podría admitir nada de la Escritura, pues, para hacerlo necesitaría primero conocer las demostraciones filosóficas, algo que claramente no puede hacer. ${ }^{31}$ Con su método, en cambio, el vulgo no requiere más que el conocimiento de la lengua hebrea. Así, el método de interpretación que propone Spinoza puede dirigirse también al vulgo, sin que ello implique que éste pueda hacer demostraciones de gran complejidad. No obstante, teniendo en cuenta que los principios señalados anteriormente implican un ordenamiento metódico, análogo al que debe seguir la investigación racional de la naturaleza, podemos afirmar no solo que el vulgo interpreta las Escrituras de acuerdo con un orden racional, sino que también necesita mantener la coherencia lógica entre las afirmaciones. Por esto, no nos parece extraño pensar que el vulgo que interpreta los textos sagrados tiene que proceder, en cierto modo, demostrativamente. En este sentido, refiriéndose al dogma de la obediencia, Spinoza afirma lo siguiente:

Como este mandamiento [es decir, el mandato de Dios de amar al prójimo] es sumamente claro y todo el resto puede ser lógicamente deducido de ese único fundamento por la simple razón, que cada cual

30 Zac, Spinoza et l'interprétation de l'Écriture, p. 126.

31 TTP, p. 215, G III 114. 
juzgue cómo ha podido suceder que hayan surgido en la Iglesia tantas disensiones y si las causas han podido ser otras que las indicadas al comienzo del capítulo VII.

Dicho en pocas palabras, el vulgo no debe ocuparse de demostraciones metafísicas complejas, aunque ello no implica negar que proceda racionalmente. En efecto, la interpretación de los textos sagrados necesariamente comienza con el principio más general y sólo desde allí puede deducir todo lo demás utilizando el conocimiento histórico disponible, tratando de evitar con las herramientas metodológicas cualquier clase de prejuicio. Pues justamente la falta de método y la interpretación prejuiciosa son la causa de todas las discrepancias religiosas.

\section{Conclusión}

Para Spinoza, la lectura de la Escritura, utilizando el método hermenéutico, en la medida en que no hay allí una extrapolación conceptual de un ámbito a otro, no conlleva una intromisión de la razón en el ámbito de la fe. Al contrario, tal método busca preservar intacto el contenido original de los textos. Dicho de un modo resumido, la aplicación de la razón consistiría en detectar las doctrinas más generales, claras y distintas, que ofrecen las Escrituras y entrelazarlos deductivamente con otras enseñanzas menos generales y a la vez menos evidentes que también están presentes en las mismas, pero sin una conexión clara. Al mismo tiempo, esta ordenación permitiría eliminar las enseñanzas que se contradicen entre sí o contradicen el núcleo principal. De este modo, se podría decir, salvando importantes diferencias -como el andamiaje metodológico, o los contenidos y fines propios de cada ámbito- que Spinoza pretende, en un sentido laxo, "geometrizar" la propia Escritura. En efecto, como hemos visto, de un modo similar a los Elementos de Euclides, como resultado del proceso de interpretación, el lector de la Biblia se encontraría, al final, con todo el contenido ordenado de manera tal que al comienzo se hallaría lo más fácil de conocer, lo más evidente, y de allí se derivaría lo menos conocido. 
Así, Spinoza parece pensar el dominio de las Escrituras -que no es otro que el de la religión- como separable de la filosofía de una manera análoga a como se pueden separar los dominios del conocimiento dentro de la filosofía. Esto es, así como se puede delimitar el ámbito de la geometría y el ámbito de la ética y sería absurdo interpretar el uno en función del otro, aplicando conceptos de la geometría a la ética, aunque claramente se pueda aplicar a ambas el mismo método, también se podría delimitar el ámbito de la religión y el ámbito de la filosofía, aplicando en ambos casos el mismo método, es decir, la razón. De este modo, para Spinoza, aplicar la razón no es mezclar los conceptos de un dominio con los del otro, sino más bien ordenar y conectar los conceptos de cada dominio de una determinada manera, esto es, siguiendo un método.

A simple vista, puede verse que la empresa no carecerá de problemas, pues, mientras que los dominios del conocimiento dentro de la filosofía pueden separarse en virtud de sus diferentes objetos, no parece ocurrir lo mismo con el caso de la religión y la filosofía cuyos temas se entrecruzan. Sea como sea, cómo responde -o cómo respondería- Spinoza a este y otros problemas similares es una cuestión que no podemos abordar aquí. Nuestro objetivo se limitaba a clarificar el modo en que Spinoza entiende la relación entre la razón y la fe en su método hermenéutico, de manera que con lo dicho ya hemos arribado a nuestro destino.

Recibido: 08/2017; aceptado: 12/2017 